\title{
Kontrolle löst Schweregrad ab
}

$D^{i}$ ie aktuellen Asthma-Empfehlungen der Deutschen Gesellschaft für Pneumologie setzen die internationalen Empfehlungen um, berichtete Prof. Dr. Helgo Magnussen, Großhansdorf: Entscheidend für die Therapiestrategie ist nun nicht mehr der Schweregrad, sondern das Maß der Asthmakontrolle. Bei einem ungenügend kontrollierten Asthma muss die Therapie eskaliert werden, z. B. durch Erhöhung der Dosis inhalativer Glukokortikoide oder durch Kombination verschiedener Dauermedikationen. Beim schwer zu behandelnden und refraktären Asthma ist auch der IgE-Antikörper Omalizumab (Xolair) von Bedeutung. Prof. Dr. Klaus F. Rabe, Leiden, betonte, dass mehr als die Hälfte dieser Patienten auch eine Allergie hat und $\operatorname{IgE}$ gerade bei allergischem Asthma eine zentrale Rolle spielt. Eine Omalizumabtherapie in Ergänzung zu anderen Dauermedikationen kann die Zahl schwerer Exazerbationen und die Notwendigkeit der Behandlungen in der Notfallambulanz senken, aber auch helfen, den Verbrauch an inhalativen Glu-

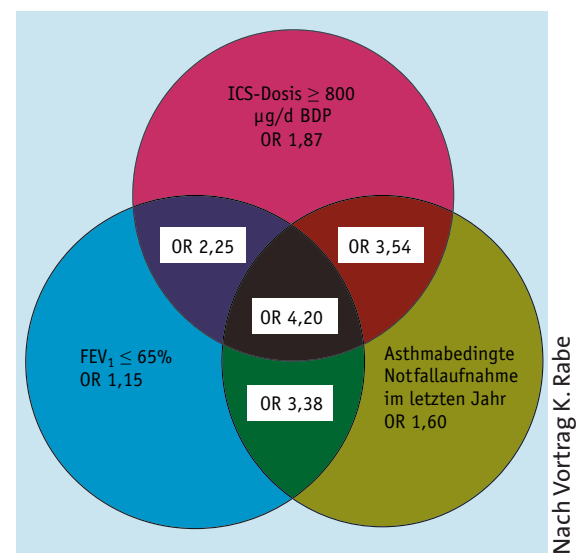

Drei Faktoren helfen, die Wahrscheinlichkeit für ein Ansprechen auf eine Anti-lgE-Therapie abzuschätzen.

kokortikoiden zu senken, berichtete Rabe. Das Ansprechen auf die Therapie lässt sich dabei in einem gewissen $\mathrm{Maß}$ durch drei Faktoren vorhersagen: ein $\mathrm{FEV}_{1} \leq 65 \%$ vom Sollwert, eine asthmabedingte Notfallaufnahme im letzten Jahr sowie eine Dosis inhalativer Kortikosteroide von $800 \mu \mathrm{g}$ und mehr pro Tag. Sind die einzelnen Faktoren alleine nur mäßig gute Prädiktoren, so ist die Wahrscheinlichkeit des Ansprechens auf die Anti-IgE-Therapie um das 4,2-fache erhöht, wenn alle drei Faktoren zutreffen (Abb.). Dabei hat sich seit Markteinführung gezeigt, dass die OmalizumabTherapie im klinischen Alltag ebenso sicher ist wie in der Studiensituation. Nebenwirkungen sind überwiegend leichtbis mittelgradig und meist von kurzer Dauer, berichtete Prof. Dr. Roland Buhl, Mainz. Anaphylaktische Reaktionen treten selten und meist bei den ersten Injektionen kurz nach Verabreichung auf. Daher wird empfohlen, die Patienten nach den ersten drei Injektionen zwei Stunden in der Praxis nachzubeobachten. Bei nachfolgenden Behandlungen genügen laut Buhl 30 Minuten. Aktuelle Studien untersuchen jetzt die Sicherheit des IgE-Antikörpers auch bei anderen Erkrankungen wie der zystischen Fibrose und der chronischen Urtikaria. $F K$

Symposium „Anti-lgE \& SIT: Status quo und Ausblick“.49. Kongress der deutschen Gesellschaft für Pneumologie und Beatmungsmedizin e.V., Lübeck, 9.-12. April 2008 Veranstalter: Novartis Pharma, Nürnberg

\section{Neue Optionen zur Allergietherapie nutzen}

\begin{abstract}
eit Oktober 2006 steht zur kausalen Behandlung von Gräserpollen-Allergien das erste Präparat zur sublingualen Immuntherapie in Tablettenform $\left(\right.$ Grazax $\left.^{\circledR}\right)$ zur Verfügung. Der Beginn der Behandlung ist noch bis acht Wochen vor der Pollensaison möglich. Prof. Dr. Claus Bachert, Gent, präsentierte die Daten der GT-08-Studie, in der Gräserpollen-Allergiker Grazax ${ }^{\circledR}$ oder Plazebo erhielten. Der Rhinokonjunktivitis-Symptomscore war im dritten Behandlungsjahr bei sublingualer Immuntherapie um etwa ein Drittel (Median: $-37 \%$, Mittel: $-29 \%)$ niedriger als in der Plazebogruppe. Der Medikamentenbedarf zeigte einen noch deutlicheren Abfall in der Verumgruppe gegenüber Plazeboeinnahme (-60\% bzw. $-40 \%)$. Während die IgG-4-Spiegel unter Plazebo nahezu konstant blieben, nahm IgG-
\end{abstract}

4 über den dreijährigen Behandlungszeitraum deutlich zu. IgE-Antikörper verringerten sich in der Verum-, nicht aber in der Plazebogruppe. An Nebenwirkungen zeigten sich lediglich lokal begrenzte Symptome wie Juckreiz oder leichte Schwellung im Mundbereich.

Nicht nur Pollen- sondern auch Milben-Allergien sollten therapeutische $\mathrm{Be}$ achtung finden. Zwar sind $80 \%$ der Monoallergien gegen Pollen gerichtet, so Prof. Dr. Hans-Christian Bergmann, Berlin, aber immerhin 30\% der Patienten mit mehr als zwei klinisch relevanten Allergien sind gegen Pollen und Milben sensibilisiert. Das Risiko für ein späteres Asthma ist bei Kindern mit „Indoor-Allergien" gegen Milben und Katzenhaare am größten. Bergmann machte auf eine plazebokontrollierte Doppelblindstudie [Blumberga et al. Allergy 2006; 61: 843-
8] aufmerksam, in der 54 Patienten mit leichtem bis mittelgradigem allergischen Asthma durch Hausstaubmilben drei Jahre mit ALK depot SQ oder Plazebo behandelt worden waren. Zum Studienende hatte sich die Einnahme von inhalativen Glukokortikoiden in der Verumgruppe um $90 \%$ gegenüber der Plazebogruppe reduziert. Der therapeutische Effekt galt auch für Patienten mit multiplen Allergien. Bergmann empfahl, für die Indikation zur spezifischen Immuntherapie eine klare Symptomatik bei Pollen über zwei Jahre und bei Milben über ein Jahr vorauszusetzen. Bei nachweisbarer bronchialer Hyperreagibilität sollte der behandelnde Arzt dagegen unmittelbar mit der Behandlung beginnen.

Symposium: „Neue Entwicklungen in der kausalen Allergie-Therapie“ anlässlich des 49. DGP-Kongresses Lübeck, 9.-12. April 2008 Veranstalter: ALK-Scherax, Wedel 